\title{
Index
}

'Abbasid era/caliphate 51, 54, 56-6o, 69, 93-95, 194

'Abd al-Malik, caliph 74-75, 86, 91, 95

Abu Bakr, caliph 86, 228

Abu Ubaydah 93

Achaemenid(s) 71, 165, 169, 182, 184-185,

189, 191, 196, 198-199, 200-204,

207, 208-210, 212-218, 220; see also

Artaxerxes III, Cyrus, Darius I,

Xerxes I

Acts of the Apostles 99-100, 104-105,

$114-115,121,134-136,234$

Afghanistan 49, 89, 204-206, 208, 210-211, 215-217

Ahriman see Angra Mainyu

Ahura Mazda, deity 13, 188-204, 206-221

Akchakan-kala 213

Alexander the Great 62, 90, 148, 169, 200, 203, 212

Alexandria 88-89, 134, 136

al-Hijr 84

al-Hira 63-64

al-Muqaddasi 78

al-Rabadha 84

al-Ramla 91

al-Walid, caliph 91, 92

al-Yahudu tablets 150, 162

American Indians see Native Americans

Amu-Darya, river 205, 209, 211-214

Anatolia 52, 62, 64, 69, 71, 75, 94, 104, $110,134-135,137-138,139,141-142,165$, 170-172; see also Ephesus

Andronovo culture 211-212, 272n84

Angka Malaya 214

Angra Mainyu 13, 190-191, 200

Antioch (Antakya) 92, 104, 110, 134-136, 141-142

Antioch (southern Anatolia) 135-136

Appiah, Kwame Anthony 5

Arab identity 51, 60-61, 67, 74, 86-87

Arabian/Persian Gulf 8o-81, 213
Arabian Peninsula/Arabia 48-50, 52-54, 56, $62-69,70-73,75-78,79-85,86,88,96$; see also Hadramaut, Hejaz, Yemen

Arabic language 56

Arad 153

Arafat 79

Aramaic 99, 107, 181, 192, 196, 202

archaeology 8, 10-12, 29-32, 40-43, 50, $66,86-95,74,79-85,121-132,138-143$, 174-184, 208-217

Ardashir, king 200

Aristotle 193

Artaxerxes III, king 201-202

Asherah, divine consort 149, 174

Assyria/Assyrians 62, 148, 152, 154-156, 158-160, 175-176, 181, 185, 203-204

Astarte, deity 174

Athanasius, bishop 105

Avesta 189-190, 192-199, 200, 202, 208, 201, 212, 218-220, 230, 232; see also Gathas

Avestan language 189, 192-204, 206-208, 201, 212-220

Axum see Ethiopia

Ayla 91

Baal, deity 174, 191

Babylon 65, 137, 147, 150, 160, 161-165, 170-172, 191

Babylonians 146-187

Badr, battle 53

Baghdad 51, 54, 62, 95, 194

Baha'í 231

Barton, Elizabeth 224

Battle Creek 43-44

Bede, the Venerable 60, 102

Beersheba 175

Behistun 201, 214

Beit Shemesh 92

Ben Sira 150

Benjamin, region/tribe 152, 159-160, 173, 175,183 
Beth Shean 92, 184

Bethany 120, 121

Bethel 175

Bethlehem 102, 118, 121, 156

Bethsaida 125-126

Bible see New Testament, Tanakh

Blavatsky, Helena 229, 232

BMAC culture 212, 215

Book of Mormon see The Book of Mormon

Buddhism/Buddhists 18, 218, 223, 230-231, 232,233

Bukhara 89

Bultmann, Rudolf 100

Burned-over District 25, 26-29, 45, 233

Byzantium/Byzantines 49, 52, 54, 61-65, 71, 79, 86-9o, 93-95, 97; see also Constantinople

Caesarea Maritima 92, 102, 110-112, 122-123, 135-136

Capernaum (Kefar Nahum) 111, 118, 119, 124,125

Central Asia 208-217

Chorasmia see Khorezm

Christianity/Christians 33, 45-46, 57 , $63-64,67,70-74,81-82,91-94,97$, $98-145,224,226$

Church of the Holy Sepulchre 127-129

Claudius, emperor 101

Clement, church father 105, 135

Codex Sinaiticus 105

Community of Christ (Reorganized Church of Jesus Christ of Latter-day Saints) 35, 228

Confucianism/Confucius 231, 232

Constantine, emperor 121, 127-128, 139-140, 143

Constantinople 51, 62-63, 89, 127

conversion, religious $18,48,49,51-52,54$, $67,70,72,74,87,90,94,97,104,116,132$, 194

copyist errors 8, 51, 101, 105, 152, 194

Corey, New York State 32

critical studies 4, 50, 96, 99, 157, 170, 222, 234

crucifixion 128

Ctesiphon 62, 71, 88, 89

Cumorah Hill 20, 22, 30

Cyrus, king 62, 65, 150, 160, 163-164, 165, 174, 191, 201, 203

Cyrus Cylinder 150, 165, 191
Dahan-e Ghulaman 216

Damascus 51, 88, 91, 93, 135-136, 171-172

Daniel, book 157, 192

Darius I, king 62, 169, 200-203, 208, 214, 218

David, king 72, 108, 118, 148, 159, 169, 174, 176

de Botton, Alain 5

Dead Sea Scrolls 115, 139, 150

Deakin, Alfred 224

Déhès 93

deism 3

Denkard 195

Deseret 37

Deutero-Isaiah see Isaiah book/prophets

Deuteronomist 154-155

Didache 108

Diocletian, emperor 104, 139, 144

documents see sources

Donner, Fred M. 74

dualism 48, 191-192, 203

Dunhuang 194

Dura Europus 104, 141

Ed-Dur 81

Edom see Idumaea

Egypt 51-52, 62, 64-65, 71, 84, 86, 89, 90, 139, 166

Ein Gedi 150, 172, 182

Elephantine 166

Elohim, deity 149, 152, 174, 184

Elusa 64

Elvend 201

Ephesus 104, 134-136, 142

Erie Canal 19, 25, 28, 31, 231

Essenes 115, 139, 191

Ethiopia 6, 53, 66, 69, 71-72

exile, Babylonian 146-174, 186, 219, 228, 232

Exodus 148, 154, 186

Ezekiel book/prophet 155, 163-164

Ezra, book/leader 156, 165-168, 170, 181, 191

Falun Gong 231

Far West, Missouri 18, 35, 39

Fars 75

Faust, Avraham 182

Finkelstein, Israel 151

fire/fire temples 190, 199, 201-204, 208, 210, 212-218

Fremont culture 41, 43 
Fulayj 81

Fustat 89,90

Gabriel, angel see Jibril

Galilee, Sea of (Kinneret, Lake Tiberias) 92, 117-120, 124-126

Galilee (region) 100, 106, 122, 117, 119, 121, $122-126,140,158,171,173,182,184,232$

Garden Tomb 128-129

Gathas 188, 189, 191, 192, 196-199, 206-208, 210, 216, 217-220

gender 228-229

Genesis, book 56, 154-155, 159, 163, 186

Gerizim 159, 167

Ghassanids 64, 67, 71, 86, 91, 93

Ginosar (Ginnesar) boat 125

Gnosticism 107-108, 139, 191

Gosiute 41-44

gospels, canonical 46, 99-100, 101-108, 112-113, 117-120, 123, 126, 134, 229; see also Quelle source

Great Salt Lake 18, 36, 37-40, 41-44, 233

Greece/Greeks 55, 62, 134-137, 170, 182, 192-193, 200, 212; see also Alexander the Great, Macedonia

Greek, language 99, 104, 107, 109, 120, 150, 185, 192-193, 200, 229

\section{Hadid 158}

hadith $57-58$

Hadramaut 76, 83

Hanina ben Dosa 115

Haram al-Sharif see Temple Mount

Harmony, Illinois 20

Hasmoneans 109-110, 116, 122, 125, 129-130, $158-159,166,179-180,181,185$

Haug, Martin 189

Hebrew, language 99, 150, 153, 155, 185

Hejaz (Hijaz) 65-67, 69, 71, 76-79, 83-85, 232; see also Mecca, Medina

Helena, empress 121, 127, 143

Hellenistic Judaea 148, 151, 156-158, 163, $166-169,178-182$

Heraclius, emperor 64

Herod Agrippa 110

Herod Antipas 102, 110, 112, 115, 117-120, 122

Herod the Great 102, 103, 110, 112, 118, 126, 129-130, 148

Herodotus 212

Himyarites 67, 72, 80, 82
Hinduism/Hindus 13, 199, 226, 230

Homs 91

Honi ha-Ma'age 115

Idumaea (Edom) 99, 110, 173

Independence, Missouri 18, 34

Indo-European 196, 199, 204, 210-212, 218

Indra, deity 202, 218

Iran 75, 203-208, 210-213, 216-220, 231-232; see also Persians

Iraq 52, 57, 62-63, 65, 71, 89-90, 162, 231; see also Baghdad

Irenaeus, bishop 105

Iroquois 23, 29-33; see also Gosiute, Iroquois, Seneca, Shoshone, Ute

Isa see Yeshua

Isaiah book/prophets 148, 156, 163-165, 187, 191

Islam/Muslims 46, 48-61, 70, 72-79, 83-97, 190, 226, 228, 230

isnad (chain of transmission) 58

Israel (country) 117

Israel (northern kingdom) 150-152, 154-155, 156-157, 158-160, 163-165, 166, 168-169, 170, 173, 174-176, 185-186; see also Samaria

Istanbul see Constantinople

Jafnids see Ghassanids

James, brother of Yeshua 100, 108, 133, 137, 228

Jeanne d'Arc 224

Jehoiachin, king 162

Jehoiakim, king 160

Jehovah see Yahweh

Jerash (Gerasa) 93

Jeremiah, book/prophet 155, 163-164

Jericho 93, 111, 120, 172

Jerusalem 11, 14-15, 30, 53, 60, 61, 66, 71, $75,78,88,90,91-92,106,110-113,115$, 116-119, 121-124, 126-132, 133-137, 145, 146-187

Jesus of Nazareth (Jesus Christ) see Yeshua

Jewish diaspora 137, 147, 150, 232

Jewish Revolt 104, 112-115, 126, 129, 131, 132, 137

Jews see Judaism

Jibril, angel 53, 56, 60, 73, 133, 227

John the Baptist 72, 91, 101-103, 115, 119

Jordan (region) 66, 80, 90-94, 117 
Jordan river/valley 92-93, 111, 117-119, 126, 170, 172-173

Josephus, Titus Flavius 101, 102, 112, 115, $118-119,124,125,137,159$

Josiah, king 154-155, 16o, 177, 186-187

Jubail 243n 6

Judaea 99-102, 106-107, 109-113, 115, 116-121, 122-123, 126, 132, 137, 144; see also Yehud

Judah (southern kingdom) 146-187; see also Yehud

Judaism/Jews 53, 56, 70-74, 77-78, 84, 92, $97,100,106-107,110,112,115-117,120$ $126,135,140,144-145,146-187,189$, 191-192, 230

Judas, gospel of 108

Justinian, emperor 64,86

Ketef Hinnom 267n116

Khirbet Beit Lei 175

Khorezm 205, 213-214

Kirtland, Ohio 18, 34, 39

Kok-tepe 214

Koran see Qur'an

Kuizely-gyr 215

Kuntillet 'Ajrud 175

Kyzyltepa 214

Lakhmids 63-65, 80, 86

Lamentations, book 155, 164

Latter-day Saints, Church of Jesus Christ of see Mormons

Lee, Ann 25, 229

Legio 141

letters, New Testament 100, 103-109, 120, 133-134, 137, 144, 229

Levant 52, 62, 66, 69, 71, 73, 75, 82, 90-91, $94,95,97,109,135,138,154,158,160$, 169-172, 177, 182-183, 185, 200, 219, 232; see also Israel, Jordan, Palestine, Syria

Lost Ten Tribes of Israel 30

Macarius, bishop 127

Maccabeans see Hasmoneans

Macedonia/Macedonians 62, 170, 185; see also Alexander the Great

Machaerus 102

Mada'in Salih 84

Magdala 125

magi 190, 200, 201, 204, 208
Mahd al-Dhahab 85

Makkah see Mecca

Manchester, NY 19, 20, 22, 24

Manichaeism 48, 71

Marduk 150, 191

Margiana 205, 209, 212-213, 214-216

Marib 66, 67, 82

Mary, mother of Yeshua 72, 115, 124, 129, $133,139,227-228$

Maslamah (Musaylimah) 73

Matthews, Robert 29

Mazdaism 188-189, 191, 193-194, 196, 198, 199-204, 208, 214, 217, 220, 230, 232-233

Mecca (Makkah) 53, 6o, 67-68, 77-79, 85, $88,96,232$

Medes/Medean 158, 203

Medina (Yathrib) 53, 60, 63, 65, 66, 68, 72, $77-78,83,85,96,232$

Mesopotamia see Babylon, Babylonians, Iraq

Meyer, Eduard 46

Middle Persian, language 193-196, 198, 218

Mikhmoret 264n64

minimalism 9, 151, 169-170

miracles 3

Mithra, deity 196, 201-202, 218

Mizpah 166, 168, 176

Mleiha 81

Moabite stone 174

Mongols 61

monolatry 1, 73, 147, 165, 168, 186

Monophysites 73

Mormon War 37

Mormons/Mormonism 14, 16-25, 27-28, 30, 33-40, 42-47, 97, 229

Moroni, angel 20, 103, 33, 227

Moses 52, 72, 147, 155, 163, 193

Mound Builders (USA) 31

Mu'awiya, caliph 92

Muhammad ibn 'Abdullah 46, 48-49, 52-6o, 69-70, 72-74, 78-79, 85-86, 96-97, 228-229

Murašu 150, 164

Muslims see Islam

Nabataeans $66,68,84,93,119,135$

Nabonidus, king 65, 150, 165

Naqsh-e Rajab 200

Naqsh-e Rustam 200, 201 
Nasrids see Lakhmids

Native Americans 29-33, 34, 37, 40-44, 45; see also Gosiute, Iroquois, Seneca, Shoshone, Ute

Nauvoo, Illinois 18, 35, 36, 39

Nazareth 111, 115, 117-119, 123-124

Nehemiah, book/governor 156, 159, $165-167,169-170,179,181$

Neo-Babylonians see Babylonians

Nero, emperor 101, 138

Nestorians 71, 73

New Persian, language 194

New Religious Movements 4, 13, 22, 225-226, 228, 233

New Testament 99-109, 119, 133, 139, 144, 190; see also Acts of the Apostles, gospels, letters

New York State 22, 24, 26-29, 33

Newton, Isaac 103

Nippur 150, 162, 164, 172

Nivahand, battle 89

Old Iranian languages 192-193, 195-196, 201, 219; see also Avestan language

Old Testament see Tanakh

Ophel 130, 176-177, 179-180

oral literature/tradition 55-56, 58-59, 100, $106,109,117,126,152-157,194-198$, 217-218, 229-230

Orientalism 4, 50

Oxus see Amu-Darya

Pahlavi see Middle Persian

Palestine 52, 61-62, 64, 71, 90-92, 99, 110-112, 119, 137, 139, 143, 149, 158, 161, 170-172, 178; see also Galilee, Israel, Judaea, Samaria, Yehud

Palmyra, Syria (Tadmur) 66, 68, 171-172

Palmyra, USA 19, 22, 24, 26-27, 33, 39, 240n15

Parsis 189

Parthians (Arcasids) 62-63, 80, 189-19o, 195, 199-200, 202, 208, 217

Pasargadae 201

Pascal, Blaise 3

Paul, Saint see Paulos

Paulos (Saint Paul) 100, 103, 104, 106-109, $116,120-121,132-137,141-142,144,227$, 229

Pella (Fihl) 93, 111, 126-127
Pentateuch see Torah

Persepolis 200-202, 204, 209

Persian Gulf see Arabian/Persian Gulf

Persians/Persian Empire 62, 137, 149; see also Achaemenid(s), Parthians, Sasanians, Yehud

Peter, apostle 108, 125, 133, 135, 143

Phelps and Gorham purchase 23, 24, 25, 33

Philip, ruler 110, 112, 119, 126

Philistia/Philistines 111, 158

Phoenicia/Phoenicians 111, 116, 158, 170, 184

plague, Justinian 64

Pliny the Younger 138

polytheism 1, 48, 69-70, 72-73, 149, 157, 174, 196, 201

Pontius Pilatus (Pilate) 102-103, 109-110, 120-121

population size 21-24, 32, 81, 85, 92, 112, 122, 124-126, 128-129, 131, 134, 137, 140-141, 158-159, 16o, 167-168, 175-18o, 182,206

post-exilic period see Yehud

prehistory see archaeology

pre-Islamic literature 56

priests 110, 113, 154, 168, 190, 195-197; see also magi

Prophet Muhammad see Muhammad ibn 'Abdullah

prophets/prophetic tradition $47,73,149$, 155-157, 163-164, 187, 189, 222, 223-224, 227-234

Protestantism 4, 18-19, 27-29, 31, 128, 230, 234

Provo 39

psalms 149, 153, 157, 163

psychiatry 225-227

Qadisiyyah, battle 88-90

Qani' 83

Qaryat al-Faw 82

Qinnasrin 91

Quelle source 104, 109

Quirinius 102

Qur'an 51-52, 55-57, 59, 72-74, 96-97, 190, 219,229

Quraysh 53, 78-79

Rabi’a 229

Rabigh 77

Raga 208 
Ramat Rahel 92, 181-182

Rashidun see Rightly Guided Caliphs

Rastafarianism 6, 226

Rightly Guided Caliphs 51-52

Rigveda see Vedic

Rochester, New York State 29

Roman Catholic 227, 229-230

Roman Empire 63, 66, 94, 100, 102, 109, 115, $116,123,133-134,137,139-142,144-145$, $147,232-233$

Rome, city 135, 142-143

Sabaeans 66-67, 70, 80, 82

Said, Edward 50

Salt Lake City see Great Salt Lake

Samaria/Samarians 110-112, 117-119, 121, $158,159,167,172-173,183-184,268 \mathrm{n} 13 \mathrm{O}$; see also Israel

Samaritans, religious group 116, 159, 167, 185

Sana'a $57,82,88$

Sasanians 49, 52, 54, 61-65, 68, 70-71, 80, $82,85-90,94-95,97,189-192,194-195$, 198-200, 203, 208, 213, 217-218, 233

Satan 190-191, 223

schisms 13, 28, 35, 51, 107, 233-234

Scientology 226

seals 178, 181-182, 202

Second Great Awakening 17, 27

Second Temple period see Yehud

secularism 2

Selassie I, Haile 6

Seneca 23, 26, 29, 33

Sennacherib, king 160, 176

Sepphoris 111-112, 116, 118, 122-126

Seventh Day Adventists 17, 29, 229

Shabwa 83

Shahr-i Sokta 216

Shakers 25, 28, 229

Shephelah 171, 173

Sh'ia 51, 56, 234

Shinto 231

Shoshone 41-43, 45

Siddhartha Gautama see Buddhism

Sikhism 231-232

Sir Bani Yas 81

sirah 58

Sistan (Seistan) 206-209, 211, 216-217

Smith, Joseph Junior 16-21, 27, 29, 31, 34-37, $45-47,103,106,155,224$

Smith, Joseph Senior 19-20, 22, 24-25, 228
Smith, Lucy Mack 2

Sogdian/Sogdiana 194, 203, 205, 207-209, 213-215

Solomon, king 72, 78, 148, 159, 169, 185-186

Sophronius, bishop 61

sources $8-10,14,17,26,46,50,54-61$, 101-108, 134, 149-157, 162, 192-199, 234

Spiritualism 224, 229

Steward, Julian H. 41

Storr, Anthony 226

Subayta 92

Subud 226

Suetonius 101, 138

Sunni 51, 56, 234

Susa 201

Synod of Hippo 105

Syr-Darya, river 205, 209, 214

Syria 52, 53, 61, 62, 64, 71, 78, 84, 90-94, 102, 104, 110, 112, 116-117, 119, 133-137; see also Antioch, Damascus, Palmyra

Tacitus 101, 138

Tadmur see Palmyra

Ta' if $79,85,88$

Tanakh 46, 115, 146-165, 174-178, 184-187, 191-192, 198, 219-220, 224, 229, 234

Taoism 231

Tash-k'irman-tepe 214

taxes $49,52,87,93,110,123,172,182-183,190$

Tayma $65,68,84$

Tel Moza 175

Temple, Jerusalem 110, 112, 115, 119, 130-132, 147-148, 150, 156, 159, 160-162, 164-170, 174, 177-178, 185, 187, 228; see also Temple Mount

Temple Mount (Haram al-Sharif) 11, 91-92, $127,131,176-177,180$

texts see sources

The Book of Mormon 20, 24, 30, 34, 36, 46, 155, 198, 219, 229

theism 3

Thenius, Otto 129

Theosophy 229, 232

Theudas 115

Thomas, gospel of 108, 120

Tiberias, city 92, 111, 117-118, 122-126

Tiberius, emperor 102, 103

Torah 154, 192-193

trade routes $62,66,68-69,71,73,75-79$

Transcendental Meditation 226 
Trench, Battle of the 53

Trinity 13, 114-115, 146

Tripoli 89

Turkey see Anatolia

Uhud, battle 53

Umar b. 'Abd al-'Aziz, caliph 87

Umar ibn al-Khattab, caliph 84, 91, 228

Umayyad era/caliphate 51, 55-56, 58, 60, 86-95

Umm al-Jimaal 93

United States of America 21-26, 240n8

Utah 36, 37; see also Great Salt Lake

Utah Lake 36, 39, 43-44

Ute 41-44, 45

'Uthman, caliph 56, 57, 78, 91, 228

Vedic/Vedas 199, 202, 206-207, 218-219, 230

Vermes, Geza 116

Via Dolorosa 129

Vishtaspa 208, 216

Weber, Max 226
Xerxes I, king 62, 201-202

Yahweh, deity $146-165,174-178,184-187$, 189

Yathrib see Medina

Yaz culture 215-216

Yehud (Persian Judah) 146-161, 165-187, 233

Yemen 54, 57, 63, 64, 66-68, 71-72, 76, $78-79,80,82-83$

Yerkurgan 215

Yeshua 72, 96, 99-134, 143-145, 228-230

Yodfat (Yodefat) 124

Young, Brigham 33, 36, 37, 38, 40-41

Zand (Zend) 195

Zarathushtra, prophet 188-192, 195-200, 202-204, 206-208, 210-212, 215-220, 228, 232

Zealots 112, 115

Zoroaster see Zarathushtra

Zoroastrianism/Zoroastrians 12, 15, 70, 87, 188-221, 230; see also Mazdaism

Zurvanism 191 
Robin Derricourt - 9781526156198 Downloaded from manchesterhive.com at 04/26/2023 10:39:22AM via free access 